\title{
Trivium
}

Revue franco-allemande de sciences humaines et sociales - Deutsch-französische Zeitschrift für Geistesund Sozialwissenschaften

28 | 2018

Capitalisme

\section{Concepts historiques du capitalisme industriel avancé : « capitalisme organisé » et « corporatisme "}

\section{Hans-Jürgen Puhle}

Traducteur : Gilles Campagnolo

\section{OpenEdition}

Journals

Édition électronique

URL : http://journals.openedition.org/trivium/5831

DOI : $10.4000 /$ trivium.5831

ISBN : 1963-1820

ISSN : 1963-1820

\section{Éditeur}

Les éditions de la Maison des sciences de l'Homme

\section{Référence électronique}

Hans-Jürgen Puhle, «Concepts historiques du capitalisme industriel avancé : « capitalisme organisé » et « corporatisme » », Trivium [En ligne], 28 | 2018, mis en ligne le 30 juillet 2018, consulté le 08 septembre 2020. URL : http://journals.openedition.org/trivium/5831 ; DOI : https://doi.org/10.4000/ trivium.5831

Ce document a été généré automatiquement le 8 septembre 2020

\section{c) () $\Theta$}

Les contenus des la revue Trivium sont mis à disposition selon les termes de la Licence Creative Commons Attribution - Pas d'Utilisation Commerciale - Pas de Modification 4.0 International. 


\title{
Concepts historiques du capitalisme industriel avancé : " capitalisme organisé » et « corporatisme »
}

\author{
Hans-Jürgen Puhle \\ Traduction : Gilles Campagnolo
}

\section{NOTE DE L'ÉDITEUR}

Nous remercions M. Hans-Jürgen Puhle ainsi que la maison d'édition Vandenhoeck \& Ruprecht de nous avoir accordé l'autorisation de traduire ce texte pour le présent numéro.

Wir danken Herrn Hans-Jürgen Puhle sowie dem Verlag Vandenhoeck \& Ruprecht für die freundliche Genehmigung, diesen Artikel in französischer Übersetzung zu publizieren.

Révision de la traduction / Überarbeitung der Übersetzung : Anthony Andurand, Hinnerk Bruhns

\section{I.}

1 Les économistes comme les spécialistes de sciences sociales semblent largement s'accorder sur le fait que le capitalisme industriel est entré à un moment donné dans une phase avancée de son développement, tout particulièrement dans ses variantes que l'on observe en Europe de l'Ouest, en Amérique du Nord et au Japon. Que le capitalisme se situe encore à cette étape, ou qu'il l'ait entre-temps déjà dépassée et laissée à nouveau derrière lui, il se différencie à tout le moins de la phase antérieure (ou bien des phases antérieures), à laquelle on peut rattacher le début d'une première industrialisation et l'avènement de l'industrialisation massive (Hochindustrialisierung). Que cette nouvelle étape soit liée plus fortement encore que la précédente à des 
tendances vers plus d'organisation et un interventionnisme étatique accru (dans les affaires intérieures comme extérieures), ainsi que vers de nouvelles formes de défense et de conciliation des intérêts, tout cela est notamment suggéré par les concepts que l'on a inventés pour désigner cette étape :

- impérialisme, capitalisme monopolistique d'État, capitalisme monopolistique ;

- capitalisme tardif (Sombart);

- [stade] néo-mercantiliste de type Kondratieff (Schumpeter) ;

- capitalisme planifié (Sering)

- modern capitalism (Shonfield) ;

- collective capitalism (Feldman);

- political capitalism (Kolko);

- corporate capitalism (Dobb, Williams) a b;

- corporatisme libre (Heckscher) ;

- corporate pluralism (Rokkan, Maier);

- quasi-corporatism (Beer) ;

- corporatism, neo-corporatism et liberal corporatism (Schmitter et Lehmbruch notamment) ${ }^{1}$.

2 L'un de ces concepts destinés à éclairer (notamment sur le plan historique) les voies que le capitalisme industriel a empruntées au cours de son développement est celui de " capitalisme organisé ». Il fut d'abord utilisé par Rudolf Hilferding, à l'époque de la Première Guerre mondiale puis vers la fin des années 1920. Depuis le début des années 1970, des historiens l'ont développé pour en faire un concept de travail analytique et idéal-typique (ou du moins un «modèle»), débarrassé des connotations politiques qu'il avait chez Hilferding, et l'ont également utilisé dans sa variante de " capitalisme organisé avec intervention de l'État » (Wehler)2.

Par "capitalisme organisé ", on entend un système d'interactions économiques, sociales et politiques qui se caractérise essentiellement par le fait que les secteurs économiques particuliers (l'agriculture, le commerce et l'industrie, ainsi que le secteur tertiaire privé) sont étroitement imbriqués l'un dans l'autre ainsi qu'avec les agences de la puissance étatique qui réglementent, administrent et fournissent des services dans une mesure toujours plus grande. Pour autant, le principe d'appropriation privée des bénéfices $\mathrm{y}$ est fondamentalement préservé, tout comme la nature privée des investissements et des décisions en matière d'investissement; de façon générale, capital commercial, capital bancaire et capital industriel s'imbriquent de manière toujours plus étroite. Le principe d'organisation se substitue également toujours plus au principe de marché, qui était déterminant dans une économie concurrentielle capitaliste de type «classique ", tandis que l'État tend de plus en plus (même si c'est encore de manière incomplète) à diriger l'économie et à intervenir au sein de la société. Pour sa part, cette propension de l'État à intervenir de plus en plus produit en retour un effet sur les institutions politiques et sur les mécanismes de consultation des populations, ainsi que sur les formes d'organisation des intérêts privés, qui tendent à se bureaucratiser et à s'associer, et dans l'ensemble de la vie politique publique.

En conséquence, la puissance étatique n'est plus nécessairement considérée expressément comme «neutre » vis-à-vis des forces sociales, au sens que d'anciennes conceptions libérales donnaient à ce terme, et ne joue plus forcément le rôle d'agent indistinctement au service de la (ou des) «classe(s) dirigeante(s)». Le concept de capitalisme organisé n'exclut pas par avance la possibilité que la puissance étatique 
garde son autonomie ; c'est au cas par cas que l'on évaluera ses marges de manœuvre partielles ${ }^{3}$.

5 Il convient de souligner à cet égard que le concept de "capitalisme organisé » ne renvoie pas aux multiples formes que peut revêtir l'aide au développement fournie par l'État durant le passage à l'industrialisation ou au cours du processus d'industrialisation lui-même. Les tendances à l'organisation auxquelles ce concept renvoie caractérisent plutôt une action stabilisatrice et protectrice d'un type nouveau, visant à édifier des structures sur le plan institutionnel et généralement soutenue par l'ensemble des parties intéressées, quoique l'intensité de cet engagement puisse varier. L'objectif principal de cette action est de maintenir la capacité fonctionnelle de l'ordre économique existant, celui d'un capitalisme industriel déjà développé, face aux soubresauts de la croissance et de la conjoncture. De ce fait, les mesures visant à garantir cet objectif ne se bornent plus au seul secteur économique. L'imbrication toujours plus étroite des domaines de l'économie, de la société et de la politique constitue davantage l'un des principaux traits distinctifs du capitalisme organisé, que l'on peut alors regarder, au niveau de la modélisation, comme tout à fait "polyvalent » au plan politique, étant donné qu'il n'y a pas de raison à ce que les tendances liées à une organisation accrue favorisent nécessairement une seule et unique transformation possible du système politique. Une comparaison entre la façon dont les États-Unis et l'Allemagne se sont développés au $\mathrm{XX}^{\mathrm{e}}$ siècle montre que l'évolution peut s'orienter, selon les cas, vers un régime autoritaire de type fasciste aussi bien que vers le réformisme libéral-démocrate ${ }^{4}$.

6 La transition vers le capitalisme organisé renforce en effet les organes exécutifs de la puissance étatique tout autant que l'ensemble des groupements qui sont capables de défendre leurs intérêts en s'organisant en corporations et en associations d'un nouveau genre. Toutefois, la direction que cet accroissement de leur poids politique peut imprimer à l'évolution du système du capitalisme industriel dépend avant tout des configurations initiales et des rapports de force qui prévalaient au début de la transition vers le capitalisme organisé. Un capitalisme organisé dans une forme pure n'existe pas dans la réalité historique : nous n'avons affaire qu'à des manifestations à chaque fois différentes, qu'il s'agit d'analyser et de décrire concrètement, ce qui rend d'autant plus nécessaire l'élaboration d'une typologie permettant d'inventorier les différences.

7 À ce jour, les quelques chercheurs qui ont tenté de travailler pour de bon avec le concept de "capitalisme organisé » l'ont utilisé en lui apportant des nuances variées, qui reflètent en partie les lignes de force des domaines de travail restreints abordés par ces auteurs. Elles ne se contredisent ni ne s'excluent mutuellement, mais ne contribuent pas non plus à délimiter clairement et strictement ce concept. Parmi les phénomènes auxquels on a pu rattacher la transition vers le capitalisme organisé, voici ceux qui, entre autres, ont toujours été signalés ${ }^{5}$ :

8 1. La centralisation et concentration croissante de la production et du capital, qu'elle soit de nature monopolistique ou oligopolistique, résultant des soubresauts de la croissance et des évolutions de la conjoncture, ainsi que la croissance supérieure à la moyenne de la part des industries «nouvelles" (celles de la "deuxième révolution industrielle", à savoir la production électrique, la grande industrie chimique, la fabrication de moteurs) et l'imbrication des banques et du capital industriel ; 
9 2. Une séparation accrue entre propriété et prise de contrôle dans l'industrie; la montée des managers (et d'autres «cadres dirigeants»); la bureaucratisation des entreprises, la planification croissante et l'augmentation plus que proportionnelle du nombre des employés ; l'emploi de formes systématiques de management, partiellement scientifiques, la «rationalisation» et enfin le développement de nouvelles technologies ;

10 3. Croissance supérieure à la moyenne du secteur des services ;

11 4. Une organisation collective pour le règlement des conflits d'intérêts et des affrontements de classe, accompagnée d'une tendance à la bureaucratisation; une organisation (au moins en partie formelle) du marché du travail ainsi que des relations de travail ; la montée en puissance de nouveaux groupements d'intérêt; l'organisation $\mathrm{du}$ secteur agricole et son imbrication avec d'autres secteurs, ainsi qu'avec la bureaucratie de l'État, ce qui suscite en retour, à l'occasion, des formes de protestation « antimodernistes ";

12 5. Imbrication accrue des sphères socio-économiques avec celles de l'État et du politique, politique de relance ou d'expansion (dans l'armement, par exemple), protectionnisme social, développement d'infrastructures, contrôle exercé sur les monopoles, augmentation de la planification macro-économique, qu'elle soit coordonnée par le privé ou par l'État, bureaucratisation touchant de nouveaux domaines d'action, mise en place d'aides de l'État à des organismes privés, et tout particulièrement une reconnaissance (formelle ou informelle) d'une ou plusieurs organisations centrales, dans les secteurs concernés, comme partenaires dans les négociations avec la puissance étatique ;

13 6. Des tendances expansionnistes de type impérialiste;

14 7. Changements intervenant au sein de la sphère étatique, dans le rapport du législatif à l'exécutif; des tendances à une gestion de prestations (Leistungsverwaltung); une transformation des rapports entre puissance publique et puissance privée, une transformation structurelle de l'«espace public», entrelacements entre partis et pressure groups ;

8. Transformation des idéologies et des mentalités collectives;

9. Progression de la capitalisation au sein des sphères qui relevaient jusqu'ici du domaine privé, comme les loisirs, la consommation ou la famille.

17 Cette énumération des phénomènes, sous la forme d'une check list un peu fastidieuse, s'est avérée très utile pour aborder chaque cas particulier, mais elle ne saurait cependant se substituer à l'effort visant à associer de manière cohérente les facteurs particuliers et à évaluer leur importance respective au sein des processus qui ont lieu dans la société dans son ensemble. Or c'est bien ce qui manque jusqu'à présent : il n'y a pas encore de théorie satisfaisante du capitalisme organisé.

À cet égard, le concept lui-même comporte encore des limites que l'on peut clairement mettre en évidence, limites qu'ont déjà soulignées en partie, dès les premiers temps, ceux qui l'ont utilisé. Ce sont notamment (1) le flou lié à l'étendue de ce concept, qui est plutôt resté, malgré ses ambitions analytiques, un concept fondamentalement descriptif - la mise en évidence de différences plus marquées, selon les secteurs et selon des phases spécifiques, et le recours à une périodisation permettraient d'en préciser les contours; (2) la distinction encore insuffisante que l'on fait, au sein du capitalisme organisé, entre les différentes instances «organisatrices » et, 
corrélativement, la difficulté à les rattacher à des motivations, à des causes et à leurs conséquences, à des intentions et à leurs résultats ; (3) les difficultés rencontrées pour quantifier complètement certains éléments déterminants, qui ont conduit de nombreux auteurs à préférer parler - sans doute plus correctement, mais de ce fait de manière bien moins claire - d'un «capitalisme qui s'organise de lui-même" ou d'un " capitalisme de plus en plus organisé ». La question principale à cet égard consiste à déterminer les critères que le concept doit à chaque fois remplir et dans quelle mesure ils doivent être remplis pour que l'on puisse parler de quelque chose comme le « début de la transition " vers le capitalisme organisé ou de l'« avènement» de celui-ci. Une réponse à cette question ne peut être trouvée que dans le cadre d'une théorie élargie, qui établirait des priorités claires parmi les possibles indicateurs (par exemple le Produit Social Brut réalisé selon les secteurs, la part des emplois occupés par chaque secteur, la quote-part de l'État, le degré d'organisation du privé, la bureaucratisation, les fédérations, les modes de règlement des conflits, la « concertation », etc.) ${ }^{6}$.

Une décennie après que l'on a mobilisé le concept une nouvelle fois, l'impression prévaut toujours qu'il révèle et dépeint une vision d'ensemble relativement englobante, mais de façon toujours très impressionniste. Abstraction faite d'améliorations toujours possibles, cet état de fait semble pourtant constituer, pour certains objectifs déterminés, une approche plus opportune que ne le serait une analyse quantitative exacte, « réaliste », mais centrée sur une portion étroite du tableau d'ensemble. Il s'agit donc pour nous de nous demander ce que le concept de capitalisme organisé peut ou ne peut pas apporter, ce à quoi il peut ou ne peut pas servir, sur quels aspects il peut être éventuellement amélioré, ou bien s'il existe des approches alternatives plus appropriées, qui le rendraient alors superflu.

\section{II.}

À en juger par la critique que nous venons de formuler, il ne saurait s'agir d'une simple polémique sur les concepts. On ne peut en effet rester attaché à cette appellation de " capitalisme organisé " s'il est possible d'utiliser une meilleure désignation. L'enjeu réside bien cependant dans les caractéristiques, les mécanismes et les processus qui doivent être désignés par ce concept, que l'on a décrits. Il ne devrait pas être bien difficile de s'entendre si c'est bien là ce que l'on veut évoquer et si l'on dit tout à fait clairement qu'on le dénommera autrement (par exemple par les termes de «modern capitalism », "political capitalism », « corporate capitalism » ou de " capitalisme industriel avancé »). Il en irait de même si l'on parlait de "capitalisme tardif "c, bien qu'il ne soit sans doute pertinent d'utiliser l'adjectif "tardif» que si l'on est convaincu que le capitalisme doit atteindre bientôt son terme - perspective assez peu vraisemblable, ou qui reste du moins à démontrer. L'appellation de « capitalisme organisé » paraît ici plus exacte et plus souple, en ce qu'elle souligne le rôle central des tendances à l'organisation - ceci dans la mesure où il paraît plus juste, précisément en raison de l'imbrication toujours plus étroite entre les sphères publique et privée, d'envisager l'intervention de l'État non (comme chez Wehler) comme un élément à part (dans une sorte de « duumvirat $\left.{ }^{7} »\right)$, mais plutôt comme une partie intégrante de l'évolution vers le capitalisme organisé. Le terme de "capitalisme» conserve son importance dans la mesure où il rend compte, en les définissant de manière globalement claire et concrète, les principes fondamentaux et toujours en vigueur de l'ordre économique. Les 
tentatives visant à éviter l'usage de ce mot (pour quelque raison que ce soit) ont seulement conduit ou bien à des analogies erronées ou mal choisies, comme celle de " néomercantilisme ${ }^{8}$ ", ou bien à mettre l'accent sur des domaines plus restreints, liés à la défense des intérêts, à l'instar du corporatism et des variantes que l'on peut composer à partir de ce terme. Quant à remplacer le terme de "capitalisme organisé " par une monstruosité lexicale telle que l'«interdépendance organisée ${ }^{9}$ ", cela ne fait tout au plus qu'obscurcir encore les choses.

21 Le fait qu'il ne s'agit pas uniquement d'une querelle de mots concerne également le seul concept concurrent aussi englobant, qui est, en outre, très élaboré au plan théorique : le "capitalisme monopolistique d'État», dans le contexte de la théorie léniniste de l'impérialisme ${ }^{10}$. Quelqu'un qui ne partage pas la vision marxiste-léniniste de l'effondrement du capitalisme "bourgeois» (vision qui exclut toute possibilité de transition réformiste-évolutionnaire) ne saurait dans l'absolu accepter, aujourd'hui comme hier, l'idée de «capitalisme monopolistique d'État». Et il serait davantage enclin - s'il ne trouvait pas de terme plus adapté ou encore s'il souhaitait tout bonnement se passer de tout échafaudage théorique - à interroger le terme de " capitalisme organisé ». D'un autre côté, il semble bien que l'on ait réussi entre-temps à écarter des arguments plus anciens, que l'on pouvait encore faire valoir il y a une dizaine d'années contre l'approche formulée en termes de capitalisme monopolistique d'État, en particulier l'idée selon laquelle la puissance de l'État serait indistinctement instrumentalisée au service de la ou des classe(s) dirigeante(s) ${ }^{11}$. C'est surtout Helga Nussbaum qui a entre-temps fermement établi que l'on a aujourd'hui « dépassé cette application rigide et schématique » de la doctrine ; elle a elle-même développé en détail des recherches, qui montrent également que l'idée de "capitalisme monopolistique d'État ", une fois convenablement délimitée et rendue opératoire, ne constitue plus nécessairement un obstacle quand il s'agit de produire des résultats fructueux et nuancés. Cette approche peut même éventuellement favoriser de tels résultats, même si - point sur lequel Jürgen Kocka a récemment attiré l'attention-, lorsque l'on est réticent à établir des distinctions, les "prémisses problématiques" de l'approche léniniste sont fortement susceptibles de diminuer sa valeur explicative ${ }^{12}$.

Dans la perspective de Nussbaum et d'autres auteurs, le capitalisme organisé renvoie au fond à la même chose que le capitalisme monopolistique ou que le capitalisme monopolistique d'État, mais suit une tout autre orientation idéologique, dans la mesure où cette approche, selon elle, ignore l'«intérêt de classe " des travailleurs et se rattache, de manière apologétique ( $\mathrm{du}$ fait de sa "polyvalence politique»), à une " théorie globale à caractère réformiste ${ }^{13}$ ". Cette critique est d'ailleurs justifiée dans la mesure où l'idée de capitalisme organisé, dans une opposition délibérée à l'idée de capitalisme monopolistique d'État, envisage l'évolution et la transformation du capitalisme dans un sens réformiste comme une possibilité parmi d'autres. Cette critique repose toutefois, elle aussi, sur des malentendus si elle postule que «la société $\mathrm{du}$ capitalisme monopolistique évolue, selon une tendance de fond, vers la "démocratie"14".

Les objections formulées à l'encontre du concept de "capitalisme organisé », tel que l'avait formulé très tôt Hilferding, ne doivent pas non plus concentrer l'attention dans la discussion critique actuelle. Il est aisé de s'en prendre aujourd'hui aux présupposés erronés et aux implications politiques non démontrées de l'approche développée par Hilferding (par exemple quant à l'importance accrue du " capital financier », ou au rôle 
tenu par les cartels comme facteurs de modération des crises), mais cela n'invalide pas la construction heuristique à l'aide de laquelle des historiens ont travaillé depuis le début des années 1970 sous l'appellation de "capitalisme organisé ", construction à bien des égards différente, en partie plus globale, en partie plus neutre au plan politique, que la conception développée à l'origine par Hilferding. Même la critique formulée entre-temps par H. A. Winkler contre l'idée du capitalisme organisé distingue encore trop peu la forme «nouvelle» de celui-ci de sa forme « ancienne $»^{15}$.

En outre, si l'on fait abstraction des faiblesses et des lacunes que les partisans du concept de "capitalisme organisé » avaient admises dès le début, il n'en reste pas moins quelques points importants, développés par les critiques ultérieurs, qu'il nous faut aborder ici :

1. En général, on a dénoncé le caractère surtout descriptif et cumulatif du concept, auquel on a reproché de rester confus et imprécis et de regrouper indistinctement, sous le terme d'" organisation », de trop nombreux phénomènes dont on serait bien en mal de fonder l'unité théorique ou conceptuell ${ }^{16}$. Cette critique est très largement justifiée. Mais il reste à examiner jusqu'à quel point des améliorations sont possibles. Et il en va de même pour tous les points ci-dessous : il y aurait toute nécessité à leur apporter, pour partie, des compléments et, pour partie, une plus grande clarté.

2. On a reproché au concept de capitalisme organisé d'avoir abordé essentiellement, jusqu'ici, les débuts de celui-ci, et guère son développement ultérieur ${ }^{17}$, d'être un concept "statique ", tout particulièrement pour cette raison qu'il ne dirait rien de ce qui viendrait après le "capitalisme organisé » ${ }^{18}$. Pour Lénine comme pour Hilferding, c'était de toute évidence le "socialisme " (même si c'était un socialisme différent pour chacun d'eux); Schmitter lui-même semble assez convaincu qu'au siècle du corporatisme devait succéder le siècle d'un "syndicalisme » à petite échelle et organisé de manière plus souple ${ }^{19}$. Lehmbruch propose en tous cas deux alternatives possibles : d'un côté, le "syndicalisme »; de l'autre, le rétablissement " néo-libéral » (au fond, de type monétariste) de la concurrence ("plus de marché!»), la déconstruction des tendances à l'organisation et une dissociation entre État et demandeurs ou offreurs libres ${ }^{20}$.

En outre, il faut noter que les partisans du concept de capitalisme organisé (peut-être parce que ce sont pour la plupart des historiens) n'ont jamais indiqué jusqu'à présent ni les possibilités ni les perspectives à cet égard. Tel que ce concept a été défini, on ne peut parler de mise en œuvre du capitalisme organisé que lorsque les organisations de travailleurs sont elles aussi reconnues comme des partenaires dans la négociation des conventions collectives et intégrées dans le système, et que les relations professionnelles sont règlementées selon une procédure associant l'État ${ }^{21}$. Dans certains pays, cette situation coïncida chronologiquement, parfois aussi au hasard des motivations et des porteurs de ces actions, avec la mise en pratique (tentative) de conceptions keynésiennes touchant le pilotage global de l'économie et le deficit spending de l'État, et ceci en partie déjà avant la formulation de ces concepts par Keynes ${ }^{22}$. En conséquence, le keynésianisme et la politique économique d'inspiration keynésienne ont été compris jusqu'ici - sans préjudice de leur importance éventuelle en tant que critères de périodisation "infra-typologiques $»^{23 \mathrm{~d}}$ - non comme le début d'une ère nouvelle à la suite du capitalisme organisé, mais davantage comme une phase - qui n'entre certes pas nécessairement dans la définition du concept, mais appelée à commencer un jour - durant laquelle l'intervention et le pilotage économiques de la 
part de l'État sont explicitement soulignés plus fortement que précédemment. Les éléments caractéristiques de cette phase demeuraient de ce fait cohérents avec la description du système du capitalisme organisé dans son ensemble, ou dans sa plus large partie, tel qu'il existait auparavant et auquel le keynésianisme fournit, pour ainsi dire, une consécration théorique ultime. À cet égard, il n'est pas faux de considérer l'installation de ce qu'on appelle le "Keynesian Welfare State " ", comme le produit final de l'ère du capitalisme organisé.

L'aube qui doit succéder au "Keynesian Welfare State" reste vague, mais le keynésianisme et l'État-Providence sont en crise depuis les années $1970^{24}$ : la forte stagflation de ces années-là, qui débuta avec la pénurie de l'énergie et des matières premières et accompagna une troisième révolution industrielle (la révolution électronique), a montré les limites non seulement de la croissance, mais aussi des capacités, de la mobilité et de l'efficacité des réseaux au sein de la sociétéf, et notamment des grandes organisations et des institutions bureaucratiques. Pour remédier à cette crise, les nouvelles recettes, souvent monétaristes, prônent alors la voie qui consiste à se détacher de l'État pour se tourner vers le marché, la fin des interventions de l'État, des systèmes d'assurances sociales universelles et des subventions gigantesques, ainsi qu'un retrait (relatif) de l'État hors de l'économie et de la société à travers la " déréglementation ». Laissons de côté la question de savoir dans quelle mesure ces recettes peuvent être appliquées ou sont appliquées. Aujourd'hui encore, on ne saurait dire si le progrès des tendances favorables à des formes d'organisation de petite taille ou aux courants de pensée préconisés par des mouvements de protestation nouveaux, issus de la base, autant que par des gouvernements conservateurs, marquent le début d'une tendance séculaire ou seulement un intermède temporaire de réactions "antimodernistes ", qui pourraient connaître un destin analogue à celui des populistes américains qui, en leur temps, ouvrirent la voie, sur le long terme, à une plus ample organisation et à un État plus for ${ }^{25} \mathrm{~g}$. Peu importe si ce capitalisme de l'ère "post-organisée " paraîtra au final effectivement plus ou moins organisé que ce qu'on appelle déjà le capitalisme « organisé »: du point de vue de l'ampleur et de l'intensité des formes dans lesquelles ces organisations s'imbriquent, ce capitalisme-ci semble bien avoir dépassé le zénith d'une évolution qui aura duré en Allemagne presque exactement un siècle. L'évolution ne semble plus s'orienter dans la même direction : l'imbrication mutuelle séculaire des secteurs économiques entre eux, comme avec l'État, ne paraît plus globalement s'accroître, bien qu'il y ait toujours eu des enclaves et des tendances contraires, durant des périodes précises, dans certains secteurs ${ }^{26}$; elle tend au contraire à se différencier et à se réordonner progressivement. Il n'est donc pas tout à fait invraisemblable que dans un futur lointain (si l'on prend assez de recul pour mieux observer le cours des événements), il puisse paraître judicieux de dater des années 1970 la fin de ce " capitalisme organisé » et de chercher un mot nouveau afin de désigner ce qui lui succède. Les historiens, cependant, ne sont pas des prophètes.

29 Si l'on pense pouvoir se tirer d'affaire en s'abstenant de caractériser conceptuellement les différentes phases du capitalisme industriel (et en se contentant donc d'ajouter des descriptions au concept de « capitalisme industriel », voire à celui, plus resserré encore, d'« industrialisation massive » (Hochindustrialisierung), pour faire valoir l'argument, certes correct, selon lequel il n'y a finalement jamais eu de capitalisme complètement inorganisé et pour attirer l'attention sur le fait que nombre des traits attribués au capitalisme « organisé » appartiennent simplement au capitalisme industriel, qui aurait 
su réagir sur avec intelligence et ingéniosité à des fluctuations et à des menaces conjoncturelles déterminées sur le long terme ${ }^{27}$, on jugera sans doute tout aussi peu nécessaire d'accorder une grande importance à cette «fin » du capitalisme organisé et ce, même si l'on devait se représenter les choses sous l'angle d'un futur distant. Au final, même de nos jours, le système industriel capitaliste ne fait que réagir aux nouveaux défis, et la stagflation des années 1970 et 1980 n'est, à sa manière, que la première, de même que la Grande Dépression d'après 1873 fut la première longue période de récession après l'avènement de l'industrialisation massive (Hochindustrialisierung) sur le continent européen.

Combattre des crises exige notamment de s'organiser. C'est certainement nécessaire. Et il en allait sans aucun doute de même dès avant le "capitalisme organisé ». Pour ceux qui tiennent à caractériser différentes phases du capitalisme industriel par des concepts bien distincts et qui ont notamment utilisé pour ce faire le concept de « capitalisme organisé », il ne s'agit pas d'opposer «organisé » à « non-organisé » ou simplement de décrire un degré plus ou moins important d'organisation, ni même de rendre compte des phénomènes séparés que sont le développement des interventions de l'État, d'une part, et l'organisation autonome de l'économie, d'autre part : comme on l'a exposé plus haut en détail, il s'agit de voir l'imbrication spécifique des traits spécifiques auxquels on reconnaît l'entrecroisement complexe des relations entre divers secteurs économiques privés et avec l'État, qui a caractérisé une certaine phase avancée du capitalisme industriel dans toute une série de pays. On aura, dans l'intervalle, largement accepté le double fait que cette phase existe et que l'on a besoin d'un concept pour la désigner ${ }^{28}$.

31 3. À l'inverse, la question des « organisateurs » respectifs trouve une réponse bien plus variable. Philippe Schmitter, en particulier, a critiqué le fait que le concept de capitalisme organisé (au moins sous la forme qu'il a prise autour de 1972/1974) sousestime certains changements fondamentaux et néglige des imputations causales nécessaires et majeures. Selon lui, le concept ne clarifiait pas vraiment la question de savoir si l'État réagit seulement à la manière dont les choses s'organisent dans le privé, ou s'il est au contraire actif à chaque fois lui-même en tant qu'organisateur. Dans le modèle du capitalisme organisé qui est proposé, on ne peut, selon Schmitter, distinguer les tendances de long terme vers une organisation « sociale " consensuelle, volontaire (une " corporatisation ${ }^{\mathrm{h}} »$ ), et la forme autoritaire d'organisation que l'État impose par le haut (le plus souvent après des ruptures majeures) ${ }^{29}$. Ce qui est demandé ici, c'est une typologie d'un niveau inférieur qui permet de différencier, typologie analogue à la distinction schmittérienne entre societal corporatism et state corporatism ${ }^{i}$ (ou encore une distinction à la Lehmbruch entre corporatisme libéral et corporatisme autoritaire ${ }^{30}$ ) l'objectif étant de ne plus faire usage d'un seul et même concept pour désigner à la fois le système national-socialiste et le New Deal, l'Espagne franquiste et la République Fédérale d'Allemagne ${ }^{31}$. Il est sans doute possible de concevoir une typologie qui permet de telles différenciations. Le concept de «capitalisme organisé » ne l'exclut en aucune manière, comme le montrent les comparaisons des évolutions en Allemagne et aux États-Unis formulées entre-temps dans le cadre de ce concept ${ }^{32}$.

4. Certains critiques ont également dénoncé le fait que le "capitalisme organisé » ne tiendrait pas compte des influences et des implications au plan international, qu'il ne dirait rien, en particulier, du rapport entre « capitalisme organisé » et impérialisme, de la divergence entre «centre » et "périphérie " (pour utiliser les termes de Sombart), 
ou du développement d'un capitalisme dépendant, "déformé ", dans les pays de la périphérie (et peut-être d'autant plus "organisé", pourrait-on ajouter, fût-ce de manière singulière ${ }^{33}$. On peut aisément combler cette lacune. Il y a dans l'intervalle des indices allant nettement dans ce sens ${ }^{34}$.

5. Au plan historique, il est assurément tout à fait exact de formuler l'objection, mise en avant de multiples côtés, selon laquelle le « capitalisme organisé » est un concept très allemand, c'est-à-dire un concept qui a été essentiellement développé à partir de l'exemple allemand ${ }^{35}$. Car il est exact que l'Allemagne a de toute évidence été le pays où le processus conduisant à renforcer l'organisation du système capitaliste s'est d'abord fait jour. Les théories concernant l'industrialisation se sont de même très longtemps appuyées sur l'exemple pionnier fourni par l'Angleterre. La genèse unilatérale du concept n'exclut cependant pas non plus qu'on le transpose à des latecomers en matière de "capitalisme organisé ", comme on a pu le démontrer entre-temps pour quelques $\mathrm{cas}^{36}$. La définition idéal-typique des critères présente ici des avantages. Elle permet des périodisations relativement claires, en fonction du début et de la fin de la transition vers le "capitalisme organisé »; elle fournit encore expressément de l'aide dans la recherche des coupures historiques correspondantes. Elle peut encore faciliter la comparaison historique et servir à dépister des "équivalents fonctionnels " dans les comparaisons $s^{37}$. Ce point fort est contrebalancé par une faiblesse notoire : sous sa forme présente, ce concept n'aide guère à établir des périodisations au sein de l'époque qu'il désigne $^{38}$. C'est par des questionnements supplémentaires que l'on doit établir ces critères de périodisation. On pourrait dire, en forçant un peu le trait, que la grille de lecture du « capitalisme organisé » s'est prêtée à l'effort de périodisation de la phase de la transition vers le capitalisme organisé, mais qu'elle n'a que rarement abordé le capitalisme organisé une fois qu'il s'était totalement épanoui.

6. Quoi qu'il en soit, le concept de "capitalisme organisé » semble mieux se prêter à l'interprétation de l'histoire allemande des cent dernières années que ceux de "pluralisme ", de " corporatisme » ou de tout autre concept lancé plus récemment. En dehors des avantages et des inconvénients signalés çà et là, ce concept offre un avantage que l'on a trop peu pris en compte dans les débats en Allemagne : en raison de son étendue notamment, qui doit être impérativement abordée, il permet d'apprécier de façon juste et exacte le mélange de "modernité » et de "retard", si important et spécifique dans le cas allemand, et qui semble poser problème à tous ceux qui dans l'interprétation du cours de l'histoire allemande au $\mathrm{XX}^{\mathrm{e}}$ siècle auraient préféré ne voir qu'un seul de ces deux aspects. Une grande part de la «modernité » qui fit la spécificité de l'Allemagne tenait à ces efforts d'organisation ou encore à ce capitalisme organisé, ou consistait dans leurs résultats mêmes, par exemple en ce qui concernait le développement des groupements d'intérêt, de la politique sociale et de la politique d'éducation ${ }^{39}$.

\section{III.}

Les points faibles que l'on a déjà concédés jusqu'ici sont donc en particulier (1) le flou qui entoure l'étendue de ce concept et son incapacité à établir des différences par secteur et une périodisation "interne »; (2) la distinction encore trop peu nette qu'il permet d'établir entre chaque "organisateur» en cause et la relation qui existe à chaque fois entre divers secteurs privés et l'État; (3) les difficultés qu'il continue de 
rencontrer pour quantifier de part en part et ordonner les priorités et les indicateurs. En dépit de ces faiblesses, le concept de "capitalisme organisé » a jusqu'ici été utilisé avec profit dans les enquêtes comparatives. Il a notamment fourni des critères bien fondés et agencés de manière cohérente pour structurer les comparaisons - sur ce point, la check list des éléments et des phénomènes rassemblés sous ce concept a montré son utilité. D'après les critères indiqués plus haut et utilisés pour la comparaison, la période de transition qui a mené au capitalisme organisé se situe donc en Allemagne entre le milieu des années 1870 (et non, comme on le prétend parfois, au milieu des années 1890) et la fin de la Première Guerre mondiale, ou encore la Révolution de Novembre 1918 (en tous cas elle ne se termina pas déjà avant 1914). En ce qui concerne les États-Unis, elle commence davantage avec l'organisation de l'économie de guerre durant la Première Guerre mondiale et se termine au plus tôt au milieu des années 1930, avec la réglementation des relations de travail par le New Deal ${ }^{40}$. Si les incitations conduisant à accroître l'« organisation " sont issues en général de secteurs privés particuliers au sein de l'économie, il y eut cependant également plusieurs phases durant lesquelles l'activité organisationnelle s'est déployée au travers de l'État.

D'un côté, la comparaison montre dans ces deux pays des tendances et des modèles communs, y compris dans l'ordre de leur apparition. Pour des raisons structurelles, le premier secteur où se manifestent les nouvelles volontés et tendances vers l'organisation est en règle générale le secteur agricole. En bout de processus se situe l'insertion des organisations du monde du travail au sein d'un système plus fortement organisé et ordonné, dont les formes peuvent d'ailleurs varier. Il en va autrement de certains intérêts déterminés (plus faibles), qui ne parviennent au contraire presque jamais à s'organiser. Les plus forts, les mieux organisés, ceux qui ont les chefs les plus énergiques, ont l'avantage de leur position; à la longue, la centralisation et la bureaucratisation de l'organisation ont aussi un rôle important. De l'autre côté, cependant, la comparaison fait aussi ressortir des divergences profondes entre les deux pays, très étroitement liées, on l'a souvent développé, aux principes, aux structures, aux forces et aux tendances qui sous-tendent leur développement - éléments déjà présents, au sein des sociétés en question, avant le début de la transition vers le capitalisme organisé, voire même souvent avant le début de l'industrialisation. Ces divergences touchent les dimensions, la dynamique et les spécificités des secteurs économiques privés et des groupes sociaux tout autant que les traditions, les manières de s'organiser et les instruments de l'intervention de la puissance étatique, ainsi que leur interaction avec divers groupes au sein de la sociétét ${ }^{41}$. C'est ici qu'apparaissent nettement les différences entre le développement de l'État dans une Europe continentale marquée par les traditions absolutistes et celui qui prévalait dans le monde anglo-saxon ${ }^{42}$.

Dans les comparaisons que l'on a établies jusqu'à présent, on a déjà implicitement décelé toute une série de variantes de l'évolution vers (et dans) le capitalisme organisé : des phases et des secteurs marqués par une organisation sociale autonome ou la collaboration de classes ${ }^{43}$ induite par l'État, et d'autres où prévaut l'organisation par l'État, des types de développement tantôt plus démocratiques, tantôt plus autoritaires, des formes et des degrés variés d'intégration des secteurs économiques particuliers (mais aussi du mouvement ouvrier), des phases et des rythmes de développement distincts selon les nations, différentes formes de transitions d'une phase à l'autre, des 
formes de retour en arrière dans le développement dont l'intensité peut varier (comme, par exemple, concernant le contrôle des cartels, les efforts pour revitaliser les mécanismes de marché, les échecs dans l'organisation), des ruptures, des tensions, des mouvements de protestation hostiles à l'organisation, qui peuvent indiquer, de manière plus ou moins nette selon les cas, de plus amples tendances dans la transformation du système.

De même que les nations ont emprunté des chemins différents vers l'industrialisation le concept d'«industrialisation» reste cependant indispensable pour évaluer la spécificité de chacun d'eux -, de même la progression vers le capitalisme industriel avancé ou vers un capitalisme plus fortement organisé se fit par des voies diverses. Ces différences deviennent particulièrement nettes lorsque l'on compare ces développements à l'aide de la grille qu'offre un concept idéal-typique unifié. On peut tenir compte de ces variantes - comme Schmitter puis, dans un autre contexte, Kocka l'ont demandét ${ }^{44}$ - et construire très explicitement des sous-types leur correspondant : par exemple, celui d'un système plus parlementaire et plus démocratique ou bien celui d'un système plus autoritaire, ou encore des types correspondant à des phases où prédomine l'auto-organisation de la société ou bien la régulation par l'État (la question centrale restant: «mais qui donc organise ? »). Tous ces sous-types paraissent se situer tantôt avant, tantôt après la mise en œuvre généralisée des instruments de pilotage économique keynésiens, et, une fois de plus, au-dessus ou en-dessous d'une ligne qui indique le montant à déterminer de la quote-part de l'État ou de l'investissement public, ou bien le degré d'organisation ou de bureaucratisation de la société dans son ensemble, ou de groupes particuliers auxquels on assigne certaines fonctions-clefs. Bref, il est à chaque fois possible de faire des distinctions et, lorsqu'elles ne portent pas sur une trop petite échelle, elles ont toute chance, la plupart du temps, d'être pertinentes.

39 Arrivé à ce point, on aura assurément également à différencier des catégories, et ce en fonction des questions qui viendront au premier plan. Ce seront là les questions traditionnelles des historiens du social et de l'économie, à savoir : Que font les agents économiques? Que fait l'État dans le capitalisme industriel avancé, et pourquoi ? Quelle forme revêt l'interaction sociale? Quels sont les rapports entre la puissance publique et les secteurs privés? Quelles nouvelles formes mixtes apparaissent ? Etc. Ces questions exigent une mise au point différente par rapport, par exemple, aux questions des sciences politiques portant sur les fondements de la légitimité, sur les opportunités de contrôle et de participation, ou sur les rapports optimaux entre organisation et spontanéité pour réaliser des objectifs déterminés, etc. ${ }^{45}$ Dans tous les cas, il y aurait à enquêter, à tous les niveaux de questionnements de ce genre, sur les divergences souvent tout à fait importantes qui existent entre les motivations et les intentions, d'une part, les résultats et leurs conséquences, d'autre part, divergences quiinsuffisamment prises en considération - constituent souvent le seul motif de vastes débats sur les imputations causales.

IV.

Ce n'est peut-être pas une coïncidence si le concept de " corporatisme » ("corporatism " ) exerce, depuis le milieu des années 1970, tout particulièrement sur les spécialistes de sciences politiques, une fascination bien supérieure à celle que suscite le concept de 
" capitalisme organisé ». Tout d'abord, il s'avère moins difficile à intégrer à l'univers conceptuel des sciences sociales nord-américaines, où le concept de « capitalism " paraît toujours, au moins en partie, être marqué «à gauche». La raison est surtout que le corporatism est défini de manière plus précise et peut ainsi être transféré plus simplement d'une arène politique dans une autre. Il s'applique encore à coup sûr au présent et il est utilisé dans la comparaison, la plupart du temps, pour l'histoire du temps présent depuis 1945, pour laquelle il existe des collections plus larges d'indicateurs sociaux permettant des quantifications. Même si, en ce domaine, le choix des indicateurs peut souvent paraître arbitraire (ou fortuit), cette ligne argumentative donne néanmoins l'impression d'une grande exactitude quand il s'agit de questions portant sur une petite échelle ${ }^{46}$.

Le concept de " corporatisme », avec des connotations néo-corporatistesk , avait déjà été proposé dès le milieu des années 1930 par Mihail Manoïlescu (qui a aussi contribué à élaborer les théories économistes réformistes de développement du Tiers Monde, en particulier le "cépalisme ${ }^{1}$ " en Amérique latine) pour désigner un phénomène fondamental de ce siècle ${ }^{47}$. Philippe Schmitter a ainsi redéfini le concept en 1974, en le distinguant des modèles du " pluralisme », du « monisme » et du " syndicalisme » :

"[...] a system of interest representation in which the constituent units are organized into a limited number of singular, compulsory, noncompetitive, hierarchically ordered and functionally differentiated categories, recognized or licensed (if not created) by the state and granted a deliberate representational monopoly within their respective categories in exchange for observing certain controls on their selection of leaders and articulation of demands and supports ${ }^{48}$."

Puisque que le concept, bien qu'il se limite clairement aux domaines de l'expression et de l'organisation des intérêts, paraissait encore trop large, on l'a depuis encore affiné différemment. Schmitter a remplacé les mots «interest representation» par "interest intermediation » et voudrait aujourd'hui limiter le concept aux types d'interactions systémiques qui visent la médiation ou la conciliation des intérêts dans un système tripartite formé par deux fédérations en conflit au sein de l'organisation sociale des intérêts et l'État en tant que médiateur ${ }^{49}$. Le corporatisme "autoritaire", le "state corporatism » dans le style fasciste, «à la Salazar » ou "à la Franco ", a alors été établi comme objet d'enquête tout à fait spécifique ${ }^{50}$. Pour désigner le concept moins autoritaire de "societal corporatism» (au sens de la définition schmittérienne la plus récente), il semble que le "liberal corporatism " (Lehmbruch) ou le néo-corporatisme (chez Beyme) s'imposent à présent ${ }^{51}$. La présence des fédérations et la relation tripartite sont définies comme des éléments essentiels de cette classification, tandis que le degré d'organisation et le monopole de la représentation des intérêts servent avant tout de paramètres. L'attention s'est portée essentiellement sur le rôle des partis et les mécanismes de régulation dans les relations de travail. Pour Klaus von Beyme, le " néo-corporatisme " constitue un système déterminé, issu d'une "forme antérieure " de capitalisme organisé et limité à des secteurs spécifiques du bargaining lié à la défense des intérêts au sein de la phase du " capitalisme tardif ", système dans lequel la pressure a été remplacée en grande partie par la concertation ${ }^{52}$.

Les restrictions apportées à sa définition ont fortement réduit l'étendue de ce concept. Il s'est surtout chargé d'un sens qui n'est relatif qu'à la seule industrie, car c'est là que la relation tripartite classique se manifeste, quand l'État sert de médiateur entre des groupements d'industriels et/ou des fédérations patronales et les syndicats, lors de la régulation des relations de travail au sein de l'industrie. Dans les situations où l'État a 
seulement affaire à une unique fédération (par exemple, celle des paysans ou de l'industrie pharmaceutique) ou bien à un groupe de fédérations unilatéralement cartellisées (médecins, fonctionnaires), on n'utilisera pas plus ce qualificatif de systèmes «néo-corporatistes » que dans d'autres configurations, pour lesquelles on ne peut identifier clairement le groupement susceptible d'être le groupement leader (comme souvent aux États-Unis) ${ }^{53}$.

Le fait même que le concept de "corporatisme libéral » est étroitement lié à des groupements leaders et à la présence de la relation triangulaire, ainsi qu'à une large formalisation de la négociation et de la conciliation, et qu'il se rattache de manière prédominante à l'industrie fait qu'il est nécessaire d'avoir d'autres concepts pour aborder de nombreux domaines (voire la plupart), mais aussi pour désigner les interactions à l'œuvre au sein du système dans son ensemble. Le concept initial et plus large de corporatisme, qui intègre des variantes du type autoritaire, définies à partir de l'État, peut à l'occasion se substituer à ces concepts, mais pas complètement. Il se trouve alors essentiellement réduit aux processus liés à l'expression, l'organisation et la conciliation des intérêts : il laisse alors de côté d'importantes évolutions économiques et sociales (par exemple, la formation des classes sociales) et leurs conséquences politiques, et il n'est pas d'une grande utilité si l'on veut préciser l'état de développement socio-économique du système étudié. En outre, les tentatives de périodisation proposées jusqu'à maintenant ont eu pour effet de schématiser les choses : des systèmes «néo-corporatifs » ne se rencontrent ainsi qu'après la Seconde Guerre mondiale. Pour le corporatisme au sens large, le mot de Schmitter parait valable : «It all began during and immediately after World War $I^{54}$ ».

Dans le cadre de théories historiques ou de théories formulées à l'usage des historiens, le « corporatisme » semble présenter des défauts considérables en tant que catégorie systémique, et il ne peut rendre superflu ni le "capitalisme organisé », ni d'autres termes qui apportent des précisions à "capitalisme». Le concept de corporatisme comporte en vérité, lui aussi, toute une série de points stimulants et de conceptions qui aident les historiens qui ont commencé à l'utiliser ${ }^{55}$; cette utilité est cependant restée limitée. En particulier, le "corporatisme" n'a encore jusqu'ici pu contribuer de manière convaincante à la périodisation interne de l'ère du capitalisme industriel " organisé » (ou " avancé ») ${ }^{56}$; tout cela reste vrai, même si, fondamentalement, rien ne s'oppose à l'idée qu'il existe des éléments (plus ou moins) corporatistes, des soussystèmes ou des phases au sein du "capitalisme organisé», et quoique les transformations quantitatives et qualitatives qui affectent la gestion des crises économiques, la régulation institutionnelle des conflits, le pilotage global et la " concertation »- éléments qui entrent en ligne de compte quand il s'agit de fournir des critères de périodisation -, aient également été abordées par la recherche sur le corporatisme.

Malgré la multiplicité des concepts mentionnés au début de cet article, tout se passe comme s'il n'était pas facile d'élargir l'offre de concepts théoriques - ou orientés par une théorie - qui soient précis et utiles à l'historien qui veut rendre compte du capitalisme industriel avancé. 


\section{BIBLIOGRAPHIE}

Abelshauser, W. (1981) : « Korea, die Ruhr und Erhards Marktwirtschaft », Rheinische Vierteljahresblätter, 45, p. 287-316.

Alber, J. (1982) : Vom Armenhaus zum Wohlfahrtsstaat, Francfort-sur-le-Main.

Alemann, U. v. (dir.) (1981) : Neokorporatismus, Francfort-sur-le-Main.

Bade, K. J. (1975) : «Organisierter Kapitalismus », Neue Politische Literatur, 20, p. 293-307.

Baran, P. A. / Sweezy, P. M. (1967) : Monopolkapital, Francfort-sur-le-Main ;

éd. anglaise : Monopoly capital, New York, 1966 ; ed. française : Le capitalisme monopoliste, Paris, 1968.

Barkin, K. D. (1975) : « Organized Capitalism », Journal of Modern History, 47, p. 125-129.

Baudis, D. / Nussbaum, H. (dir.) (1978) : Wirtschaft und Staat in Deutschland vom Ende des 19. Jahrhunderts bis 1918/1919, Berlin.

Beer, S. (1969) : Modern British Politics, Londres.

Bennett, D. C. / Sharpe, K. E. (1981) : «Transnational Corporations, Export Promotion Policies and US-Mexican Automotive Trade », The Wilson Center Latin American Program, Working papers, $\mathrm{n}^{\circ} 104$, Washington.

Berger, S. (dir.) (1981) : Organizing Interests in Western Europe, Cambridge.

Beyme, K. v. (1979) : « Der Neokorporatismus und die Politik des begrenzten Pluralismus in der Bundesrepublik », in : Habermas, J. G. (dir.) : Stichworte zur « Geistigen Situation der Zeit », Francfort-sur-le-Main, p. 229-262.

Beyme, K. v. (1984) : « Der Neokorporatismus - Neuer Wein in alte Schläuche? », Geschichte und Gesellschaft, 10 (2), Göttingen, p. 211-233.

Bombach, G. et al. (dir.) (1976) : Der Keynesianismus, 2 vol., Berlin.

Borchardt, K. (1982) : Wachstum, Krisen, Handlungsspielräume der Wirtschaftspolitik, Göttingen.

Brown, E. H. P. / Browne, M. H. (1968) : A Century of Pay, Londres.

Cardoso, F. H. / Faletto, E. (1971) : Dependency and Development in Latin America, Berkeley.

Cawson, A. (1983) : «A Preliminary Bibliography of Modern Corporatism », Sussex Working Papers on Corporatism, Brighton.

Cox, A. / Hayward, J. (1983) : « The Inapplicability of the Corporation Model in Britain and France: The Case of Labor », International Political Science Review, 4, p. 217-240.

Dobb, M. H. (1963 [1947]) : Studies in the Development of Capitalism, Londres.

Dominguez, J. (1983) : The International Implications of Mexico's Internal Politics, Berkeley.

Evans, P. (1979) : Dependent Development. The Alliance of Multinational, State and Local Capital in Brazil, Princeton.

Feldman, G. D. (1974) : « Der deutsche Organisierte Kapitalismus während der Kriegs- und Inflationsjahre 1914-1923 », in : Winkler, H. A. (dir.) : Organisierter Kapitalismus, Vorausetzungen und Anfänge, Göttingen, p. 150-171. 
Flora, P. / Heidenheimer, A. J. (dir.) (1981) : The Development of Welfare States in Europe and America, New Brunswick.

Flora, P. et al. (1982) : State, Economy and Society in Western Europe: 1815-1975. A Data Handbook, Francfort-sur-le-Main.

Geyer, M. / Lüdtke, A. (1975) : « Krisenmanagement, Herrschaft und Protest im organisierten Monopolkapitalismus (1890-1939)», Sozialwissenschaftliche Informationen, 4, p. 12-23.

Gründel, R. et al. (1967) : Zur Theorie des staatsmonopolistischen Kapitalismus, Berlin.

Haumann, H. (1979) : « Staatsintervention und Monopole in Zarenreich - ein Beispiel für Organisierter Kapitalismus? », Geschichte und Gesellschaft, 5, p. 336-355.

Heckscher, G. (1951) : Staten och organisationerna, Stockholm.

Heidenheimer, A. J. et al. (1983 [1976]) : Comparative Public Policy, Londres.

Hertner, P. (1978) : « Banken und Kapitalbildung in der Giolitti-Ära », Quellen und Forschungen aus italienischen Archiven und Bibliotheken, 58, p. 466-565.

Hertner, P. (1980) : « "Relative Rückständigkeit”, Währungssystem, Protektionismus und Stabilisierung der wirtschaftliche Entwicklung vor 1914: Rußland und Italien als Beispiele », in : Schüller, A. / Wagner, A. (dir.) : Außenwirtschaftspolitik und Stabilisierung von Wirtschaftssystemen, Stuttgart, p. 99-123.

Hilferding, R. (1915) : « Arbeitsgemeinschaft der Klassen », Der Kampf, 8, p. 322-329.

Hilferding, R. (1927) : « Die Aufgaben der Sozialdemokratie in der Republik », compte rendu des journées du parti social-démocrate de Kiel en 1927, Berlin, p. 165-184.

Institut für Gesellschaftswissenschaften beim ZK der SED (1968 [1965]) : Imperialismus heute, Berlin.

Institut für Gesellschaftswissenschaften beim ZK der SED (1972) : Der Imperialismus der BRD, Berlin. IPSA Study Group (1982) : Representation and Repression in Contemporary State Structures, Rio de Janeiro.

Jenkins, R. (1977) : Dependent Industrialization in Latin America: The Automotive Industry in Argentina, Chile and Mexico, New York.

Katz, F. (1981) : The Secret War in Mexico, Chicago.

Kocka, J. (1969) : Unternehmensverwaltung und Angestelltenschaft am Beispiel Siemens 1847-1914, Stuttgart.

Kocka, J. (1974) : «Organisierter Kapitalismus oder Staatsmonopolistischer Kapitalismus? » in : Winkler, H. A. (dir.) : Organisierter Kapitalismus, Vorausetzungen und Anfänge, Göttingen, p. 19-35.

Kocka, J. (1977) : Angestellte zwischen Faschismus und Demokratie, Göttingen.

Kocka, J. (1980) : « Organisierter Kapitalismus im Kaiserreich? », Historische Zeitschrift, 230, p. 613-631.

Kolko, G. (1967 [1963]) : The Triumph of Conservatism, Chicago.

Lehmbruch, G. (1979a) : «Consociational Democracy, Class Conflict and the New Corporatism », in : Schmitter, P. C. / Lehmbruch, G. (dir.) : Trends Toward Corporatist Intermediation, Beverly Hills, p. 53-61. 
Lehmbruch, G. (1979b) : «Liberal Corporatism and Party Government », in : Schmitter, P. C. / Lehmbruch, G. (dir.) : Trends Toward Corporatist Intermediation, Beverly Hills, p. 147-183.

Lehmbruch, G. (1979c) : « Concluding Remarks: Problems for Future Research on Corporatist Intermediation and Policy Making ", in : Schmitter, P. C. / Lehmbruch, G. (dir.) : Trends Toward Corporatist Intermediation, Beverly Hills, p. 299-309.

Lehmbruch, G. (1983) : «Interest Intermediation in Capitalist and Socialist Systems », International Political Science Review, 4, p. 153-172.

Lehmbruch, G. / Schmitter, P. C. (dir.) (1982) : Patterns of Corporatist Policy Making, Beverly Hills.

Love, J. (1980) : « Raúl Prebisch and the Origins of the Doctrine of Unequal Exchange », Latin America Research Review, 15 (3), p. 45-72.

Maier, C. S. (1975) : Recasting Bourgeois Europe, Princeton.

Maier, C. S. (1981) : " "Fictitious bonds... of wealth and law". On the theory and practice of interest representation ", in Berger (dir.) : Organizing Interests in Western Europe, Cambridge, p. 27-61.

Manoïlescu, M. (1929) : Théorie du protectionnisme et de l'échange international, Paris.

Manoïlescu, M. (1934) : Le siècle du corporatisme. Doctrine du corporatisme intégral et pur, Paris.

Manoïlescu, M. (1937) : Le parti unique, Paris.

Moran, T. (1974) : Multinational Corporations and the Politics of Dependence: Copper in Chile, Princeton.

Newfarmer, R. / Mueller, W. (1975) : « Multinational Corporations in Brazil and Mexico: Structural Sources of Economic and Non-Economic Power », Report to the Sub-Committee on Multinational Corporations, Committee on Foreign Relations, US Senate, Washington.

Nipperdey, T. (1975) : « Wehlers "Kaiserreich". Eine kritische Auseinandersetzung », Geschichte und Gesellschaft, 1, p. 538-560.

Nipperdey, T. (1977) : Compte rendu de Organisierter Kapitalismus. Voraussetzungen und Anfänge de H. A. Winkler, Historische Zeitschrift, 225, p. 473-476.

Nipperdey, T. (1979) : « Organisierter Kapitalismus, Verbände und die Krise des Kaiserreichs », Geschichte und Gesellschaft, 5, p. 418-433.

Nocken, U. (1978) : « Corporatism and Pluralism in Modern German History », in : Stegman, D. et al. (dir.) : Industrielle Gesellschaft und politisches System, Festschrift für F. Fischer, Bonn, p. 37-56.

Nussbaum, H. (1970) : « Zur Imperialismustheorie W. I. Lenins und zur Entwicklung des deutschen Imperialismus bis 1914 », Jahrbuch für Wirtschaftsgeschichte, IV, p. 25-65.

Nussbaum, H. (1976) : « Zur Diskussion um den historischen Platz des staatsmonopolistischen Kapitalismus in der neueren marxistisch-leninistischen Literatur », Jahrbuch für Wirtschaftsgeschichte, I, p. 69-93.

Offe, C. (1983) : « Comparative Party Democracy and the Keynesian Welfare State: Factors of Stability and Disorganization », Policy Sciences, 15, p. 225-246.

Offe, C. (1984) : « Korporatismus als System nichtstaatlicher Makrosteuerung? Notizen über seine Voraussetzungen und demokratischen Gehalte », Geschichte und Gesellschaft, 10 (2), Göttingen, p. 234-256.

Panitch, L. (1979) : «The Development of Corporatism in Liberal Democracies », in : Schmitter, P. C. / Lehmbruch, G. (dir.) : Trends Toward Corporatist Intermediation, Beverly Hills, p. 119-146. 
Panitch, L. (1980) : «Recent Theorizations of Corporatism: Reflections on a Growth Industry », British Journal of Sociology, 31, p. 159-187.

Pempel, T. J. / Tsunekawa, K. (1979) : « Corporatism Without Labor? The Japanese Anomaly », in : Schmitter, P. C. / Lehmbruch, G. (dir.) : Trends Toward Corporatist Intermediation, Beverly Hills, p. 231-270.

Pollard, S. (1984) : « Keynesianismus und Wirtschaftspolitik seit der Großen Depression », in : Geschichte und Gesellschaft, 10 (2), Göttingen, p. 185-210.

Puhle, H. J. (1973) : «Vom Wohlfahrtsausschuß zum Wohlfahrtsstaat », in : Ritter, G. A (dir.) : Vom Wohlfahrtsausschuß zum Wohlfahrtsstaat, Cologne, p. 29-68.

Puhle, H.-J. (1974) : « Der Übergang zum Organisierten Kapitalismus in den USA », in : Winkler, H. A. (dir.) : Organisierter Kapitalismus, Vorausetzungen und Anfänge, Göttingen, p. 172-194.

Puhle, H. J. (1975) : Politische Agrarbewegungen in kapitalistischen Industriegesellschaften, Göttingen.

Puhle, H. J. (1979) : « Theorien in der Praxis des vergleichenden Historikers », in : Kocka, J. /

Nipperdey, T. (dir.) : Theorie und Erzählung in der Geschichte, Munich, p. 119-136.

Puhle, H. J. (1980) : « Preußen: Entwicklung und Fehlentwicklung », in : Puhle, H. J. / Wehler, H.-

U. (dir.) : Preußen im Rückblick, Göttingen, p. 11-42.

Puhle, H. J. (1983a) : «Vom Programm zum Versatzstück. Zehn Thesen zum deutschen Konservatismus ", Kursbuch, 73, p. 45-60.

Puhle, H. J. (1983b) : « Was ist Populismus? », Politik und Kultur, 10 (1), p. 22-43.

Rokkan, S. (1966) : « Norway: Numerical Democracy and Corporate Pluralism », in : Dahl, R. (dir.) : Political Oppositions in Western Democracies, New Haven, p. 70-115.

Salisbury R. H. (1979) : « Why no Corporatism in America? », in : Schmitter, P. C. / Lehmbruch, G. (dir.) : Trends Toward Corporatist Intermediation, Beverly Hills, p. 213-230.

Schirrmeister, C. (1969) : «Staatsmonopolistischer Kapitalismus », in : Wörterbuch der marxistischleninistischen Soziologie, Köln, p. 448-452.

Schmitter, P. C. (1979a) : «Still the Century of Corporatism? (1974)», in : Schmitter, P. C. /

Lehmbruch, G. (dir.) : Trends Toward Corporatist Intermediation, Beverly Hills, p. 7-52.

Schmitter, P. C. (1979b) : « Modes of Interest Intermediation and Models of Societal Change in Western Europe (1977) », in : Schmitter, P. C. / Lehmbruch, G. (dir.) : Trends Toward Corporatist Intermediation, Beverly Hills, p. 63-94.

Schmitter, P. C. (1983) : « Democratic Theory and Neo-Corporatist Practice », Social Research, 50 (4), p. 885-928.

Schumpeter, J. A. (1964 [1939]) : Business Cycles, New York.

Senghaas, D. (dir.) (1972) : Imperialismus und strukturelle Gewalt, Francfort-sur-le-Main.

Senghaas, D. (dir.) (1974) : Peripherer Kapitalismus, Francfort-sur-le-Main.

Senghaas, D. (dir.) (1979) : Kapitalistische Weltökonomie, Francfort-sur-le-Main.

Sering, P. (= R. Löwenthal) (1947) : Jenseits des Kapitalismus, Lauf.

Shonfield, A. (1969 [1965]) : Modern Capitalism. The Changing Balance of Public and Private Power, Londres.

Skocpol, T. (1979) : States and Social Revolutions, Cambridge. 
Sombart, W. (1932) : Die Zukunft des Kapitalismus, Berlin.

Staudinger, H. (1967) : « Die Änderungen in der Führerstellung und der Struktur des Organisierten Kapitalismus », in : Festschrift für Gert von Eynern, Berlin, p. 341-373.

Tilly, C. (dir.) (1975) : The Formation of National States in Western Europe, Princeton.

Treue, W. (1960) : «Wirtschafts- und Sozialgeschichte Deutschlands im 19. Jahrhundert », in : Gebhardt, B. et al. (dir.) : Handbuch der Deutschen Geschichte, vol. 3, Stuttgart, p. 315-413.

Wehler, H.-U. (1970) : « Theorieprobleme der modernen deutschen Wirtschaftsgeschichte (18001954) », in : Festschrift für Hans Rosenberg, Berlin, p. 66-107.

Wehler, H.-U. (1974) : « Der Aufstieg des Organisierten Kapitalismus und Interventionsstaates in Deutschland », in : Winkler, H. A. (dir.) : Organisierter Kapitalismus, Vorausetzungen und Anfänge, Göttingen, p. 36-57.

Wendt, B. J. (1974) : «War Socialism. Erscheinungsformen und Bedeutung des Organisierten Kapitalismus in England im Ersten Weltkrieg ", in : Winkler, H. A. (dir) : Organisierter Kapitalismus, Vorausetzungen und Anfänge, Göttingen, p. 117-149.

Williams, W. A. (1966 [1961]) : The Contours of American History, Chicago.

Winkler, H. A. (dir.) (1974) : Organisierter Kapitalismus, Vorausetzungen und Anfänge, Göttingen.

Winkler, H. A. (1979) : « Organisierter Kapitalismus? Versuch eines Fazits », in : id. : Liberalismus und Antiliberalismus, Göttingen, p. 264-271.

Wirth, M. (1972) : Kapitalismustheorie in der DDR, Francfort-sur-le-Main.

Wulf, P. (1980) : « Wirtschaft im Übergang vom Kaiserreich zur Weimarer Republik », in :

Boockmann, H. et al. (dir.) : Geschichte und Gegenwart, Festschrift für K. D. Erdmann, Neumünster, p. 275-288.

Zapf, W. (dir.) (1974-1975) : Soziale Indikatoren, 3 vol., Francfort-sur-le-Main.

Zumpe, L. (dir.) (1976) : Wirtschaft und Staat im Imperialismus, Berlin.

\section{NOTES}

a. Les notes infrapaginales appelées par des lettres sont nos notes de traducteur, celles appelées par des chiffres sont de l'auteur. Les italiques dans le texte sont toujours de l'auteur [N.d.T.].

b. Le terme corporate capitalism renvoie habituellement principalement aux grandes entreprises; or si « capitalisme corporatiste » peut servir de calque, indiquons au lecteur de garder à l'esprit que ce terme s'applique souvent dans sa version anglaise aux entreprises, mais que l'auteur l'emploiera ici aussi en langue allemande pour désigner tout autant d'autres grandes institutions bureaucratisées que les entreprises. Une remarque similaire vaut pour le terme "pluralisme corporatiste ", qui renvoie en anglais surtout aux firmes, alors que cette notion d'organisation concerne des institutions bureaucratisées autres que les entreprises. Dans tout ce texte, l'auteur interroge la notion de structure organisée plus qu'il ne met en avant l'opposition entre firmes et agences publiques. Cet accent sur la notion d'organisation plutôt que sur cette différenciation entre firmes et institutions étatiques imprègne tout le texte [N.d.T.].

1. Concernant ces concepts, voir les travaux de l'Institut für Gesellschaftswissenschaften beim ZK der SED [Institut pour les sciences sociales auprès du Comité Central du Parti socialiste unifié d'Allemagne (de l'Est)], notamment : Institut für Gesellschaftswissenschaften beim ZK der SED (1968 [1965]) ; Institut für Gesellschaftswissenschaften beim ZK der SED (1972); voir aussi 
Nussbaum (1970) ; Baran / Sweezy (1967) ; Sombart (1932) ; Schumpeter (1964 [1939]), p. 248, 279 ; Sering (1947), p. 89-108; Shonfield (1969 [1965]) ; Feldman (1974), p. 151; Kolko (1967 [1963]), p. 57 sq., p. 255 sq. ; Dobb (1963 [1947]), p. 268 ; Williams (1966 [1961]), p. 343 sq. ; Heckscher (1951) ; Rokkan (1966), p. 70-115 ; Maier (1975) ; Beer (1969), p. 419 ; Schmitter (1979a), (1979b) ; Lehmbruch (1979a), (1979b).

2. Voir Hilferding (1915), p. 322 ; Hilferding (1927) ; ainsi que les contributions figurant dans Winkler (1974), particulièrement le texte de Wehler (1974).

3. Hormis Wehler, on trouvera plus de détails chez Kocka (1974) et Puhle (1974). Voir déjà précédemment Wehler (1970); Kocka (1969), p.315-319 et Puhle (1973). Voir également (et différemment) Staudinger (1967).

4. Voir Puhle (1975), p. 15 sq., 28 sq., 113 sq. ; Kocka (1977), p. 39 sq., 66 sq., 296 sq.

5. Des accentuations différentes se trouvent déjà dans les contributions dans Winkler (1974). L'intérêt des chercheurs s'est porté sur les mécanismes politiques de la stabilisation économique, l'organisation et les conflits d'intérêts variés, les partis et les associations, l'interaction entre l'État et la société ; cet intérêt de nature théorique a conduit à mettre au premier plan et à souligner l'intervention de l'État, le protectionnisme solidaire, l'impérialisme social, le subventionnisme, le complexe militaro-industriel et l'intégration graduelle du mouvement ouvrier organisé. Des questionnements socio-historiques davantage centrés sur la formation des classes sociales et la répartition des couches sociales ont contribué à mettre plus fortement l'accent sur l'élargissement du secteur tertiaire, du salariat (y compris les managers), du scientific management, de la bureaucratisation et de la rationalisation. La contradiction apparente entre protectionnisme et rationalisation, qui sont tous deux considérés comme caractéristiques de la nouvelle étape du capitalisme industriel, résulte d'une sectorialisation des champs sociaux discutés.

6. Cf. déjà Puhle (1974), p. 172-174 et 186-188.

c. Spätkapitalismus, le terme forgé par Werner Sombart cité au début de l'article [N.d.T.]

7. Wehler (1974), p. 48 sq.

8. Ainsi, par exemple, chez Treue (1960), p. 403 sq. et passim. Les différences majeures entre le mercantilisme et les formes développées du capitalisme industriel, que néglige le terme de «néomercantilisme» formé par analogie, me semblent résider tout particulièrement dans la différence qui existe entre la prédominance des fonctions de développement, d'une part, et des fonctions de stabilisation de l'intervention étatique, d'autre part, ainsi que dans le poids distinct accordé à la structure hiérarchique (selon la question : qui est au service de qui ?).

9. Ainsi chez Wulf (1980), p. 285 sq.

10. Voir la note 1. Pour aller plus loin: Schirrmeister (1969); Gründel et al. (1967). Voir également Wirth (1972).

11. Voir en particulier la contribution de Kocka dans Winkler (1974), notamment p. 26-29.

12. H. Nussbaum in: Baudis / Nussbaum (1978), p. 9-50 (citation p.49), ainsi que les chapitres suivants (p. 51-252). Pour une critique de ce point de vue, voir Kocka (1980), en particulier p. 621. Voir également Nussbaum (1976) et Zumpe (1976).

13. Nussbaum in : Baudis / Nussbaum (1978), p. 46-50, en particulier p. 49 sq. Voir également de manière similaire S. Dillwitz in : Probleme der Agrargeschichte des Feudalismus und des Kapitalismus, t. XI, Rostock, 1978, p. 125-146.

14. Nussbaum in : Baudis / Nussbaum (1978), p. 50.

15. Winkler (1979). Winkler se réfère tout particulièrement à sa propre interprétation antérieure du capitalisme organisé, étroitement inspirée de Hilferding in : Winkler (1974), p. 252-263. Sa proposition de parler en lieu et place de cela d'un "capitalisme industriel ", avant ou après Keynes, souligne la rareté des offres conceptuelles alternatives.

16. Voir déjà Feldman (1974), p. 152 sq. ; Schmitter (1979b), p. 84 ; Barkin (1975) ; Winkler (1979), p. 267. 
17. Voir surtout Bade (1975), notamment p. 305.

18. Schmitter (1979b), p. 85 ; Nipperdey (1977) ; Winkler (1979), p. 267, 271.

19. Schmitter (1979a), p. 42.

20. Lehmbruch (1979c), notamment p. 308. De manière plus nuancée (même en ce qui concerne l'autonomie des sous-systèmes), voir Lehmbruch (1983).

21. C'est pourquoi l'idée qu'évoque l'utilisation du concept de corporatisme par certains de ses défenseurs, que le («neo »-)« corporatisme » suivrait dans une certaine mesure au « capitalisme organisé » et correspondrait à un stade plus avancé des systèmes du capitalisme industriel, est en contradiction avec les définitions établies jusque-là.

22. Sur ce point, voir Pollard (1984) ainsi que Bombach (1976). Voir aussi Borchardt (1982), p. 165-224.

23. Voir à ce propos Winkler (1979), p. 267.

d. L'auteur évoque un niveau « infra-typologique » qui est bien son idée (et non celle de Winkler) pour désigner des critères de périodisation aptes à délimiter de façon plus exacte les uns par rapport aux autres les sous-types (Untertypen) (voire les "phases") relatifs au capitalisme (correspondance avec l'auteur, 15 février 2018) [N.d.T.].

e. Soit l'État-Providence de type keynésien : nous laissons l'expression en italiques en anglais et entre guillemets comme dans la phrase suivante [N.d.T.].

24. Voir Offe (1983); voir aussi Pollard (1984).

f. On évite ici le terme " réseaux sociaux », qui serait anachronique pour l'époque où ce texte fut écrit. Plus bas, le terme « déréglementation » traduit la présence de deregulation en anglais entre guillemets dans l'original [N.d.T.].

25. Pour plus de détails, voir Puhle (1983a), en particulier p. 58 sq; Puhle (1983b) et la contribution de Claus Offe dans le même volume. Sur l'évolution aux États-Unis : Puhle (1975), p. $142 \mathrm{sq}$.

g. L'auteur fait référence au People's Party (ou " parti populiste ») américain qui fut pour un temps la troisième force électorale aux États-Unis au début $\mathrm{du} \mathrm{XX}^{\mathrm{e}}$ siècle et qui proposa des candidats qui se retiraient en général au profit des Démocrates. De fait, ce parti se fondit dans le parti démocrate comme son aile "gauche", progressiste (au sens de la défense des intérêts des travailleurs) et institutionnaliste, privilégiant les prérogatives de l'état fédéral. Plus loin, l'auteur mesure le temps du siècle passé à l'échelle de la date de son texte (1984) [N.d.T.].

26. Winkler a par exemple attiré l'attention sur les phénomènes sectoriels liés à la dissociation partielle de l'État et de l'économie dans les après-guerres, par le biais d'une législation sur les cartels; Winkler (1979), p. 267, 269 sq. D'autres facteurs parmi ceux qu'il nomme, comme l'internationalisation de la production après 1945 et les changements des formes de conflit entre capital et travail, me semblent plutôt aller dans la direction opposée.

27. Par exemple Winkler (1979), p. 266 sq.

28. Voir la note 1. Pour ce qui concerne le « capitalisme organisé », voir aussi Nipperdey (1979), p. 418 sq.

h. Nous tentons de rendre ainsi Korporatisierung [N.d.T.].

29. Schmitter (1979b), p. 84 sq.

i. Ces termes sont en anglais dans l'original, assez transparents. L'auteur les reprend de l'usage qu'en fait Schmitter, nous les laissons inchangés et renvoyons le lecteur aux textes de ce dernier que l'auteur mentionne [N.d.T.].

30. Voir Schmitter (1979a), p. 22 ; Lehmbruch (1979a), p. 53-55 ; Lehmbruch (1979b), p. 148-151.

31. Cette critique rejoint le reproche formulé par Winkler, selon lequel le «capitalisme organisé » ne contribue qu'assez peu à l'analyse d'évolutions politiques différentes, et qu' " on ne saurait créditer le capitalisme, sur le plan conceptuel, de ce qui a dû lui être arraché sous la pression de la classe ouvrière organisée et grâce aux moyens dont dispose l'État ». Voir Winkler (1979), p. 369. 
32. Voir la note 4.

33. Voir en premier lieu Bade (1975), p. 305 et Schmitter (1979b), p. 85. Les auteurs du volume collectif coordonné par Winkler ont en tous cas interrogé les liens qui unissent le capitalisme organisé et l'impérialisme, en prenant en considération le pays impérialistes. Outre Wehler et Puhle, voir aussi Wendt (1974), notamment p. 136 sq.

34. Est représentatif de cette façon de travailler (même si les concepts théoriques ne sont pas explicités) : Katz (1981). Les prémices de cette approche apparaissent également dans divers ouvrages sur la politique de l'armement et du commerce au niveau transnational ainsi que sur les multinationales, par exemple dans la pharmacie et dans l'industrie automobile. Voir par exemple, pour le monde latino-américain : Cardoso / Faletto (1971) ; Moran (1974) ; Newfarmer / Mueller (1975) ; Jenkins (1977) ; Bennett / Sharpe (1981) ; Evans (1979) ; Dominguez (1983). Sur les questionnements liés au « capitalisme de la périphérie » et à la « nouvelle division internationale du travail », voir notamment les introductions des trois volumes coordonnés par D. Senghaas : (1972), (1974), (1979).

35. Geyer / Lüdtke (1975), p. 15 ; Schmitter (1979b), p. 84.

36. Outre les contributions dans Winkler, Organisierter Kapitalismus, et les travaux sur les ÉtatsUnis cités dans la note 4, voir en particulier les cas de l'Italie et de la Russie: Hertner (1978), (1980) ; Haumann (1979).

37. Voir sur ce point Puhle (1979).

38. Certains critiques ne distinguent parfois pas assez précisément les différentes manières d'établir une périodisation et les buts variés qu'elle vise. Voir Bade (1975), p. 307 ; Winkler (1979), p. 267 sq. ; Kocka (1980), p. 626.

39. Les traits relativement « modernes » de l'évolution allemande sont à juste titre soulignés par Nipperdey (1977); Nipperday (1975), en particulier 554 sq. Voir également Puhle (1980), notamment p. 36 sq.

j. Il s'agit de la révolution allemande qui mit fin au II ${ }^{\mathrm{e}}$ Reich [N.d.T.].

40. Voir la littérature citée dans les notes 3 et 4.

41. Ibid., en particulier Puhle (1975) et Kocka (1977).

42. Outre Puhle (1973) voir également les contributions réunies dans Tilly (1975). Récemment, également à la suite à Barrington Moore, voir l'approche de Skocpol (1979). Récemment, même la science politique nord-américaine (ou d'inspiration nord-américaine) découvre progressivement le phénomène de "Sozialdisziplinierung» («disciplinarisation sociale»; G. Oestreich). Voir les contributions de l'IPSA Study Group (1982).

43. Voir Panitch (1979), p. 135 sq. et Panitch (1980).

44. Schmitter (1979b), p. 85 ; Kocka (1980), p. 627-629.

45. Voir Beyme (1984) et Offe (1984) [contributions dans le numéro thématique « Kapitalismus, Korporatismus, Keynesianismus» de Geschichte und Gesellschaft $\left(10 / 1984, n^{\circ} 2\right)$ dans lequel le présent article de H. J. Puhle a été publié ; N.d.T.].

46. Voir entre autres les contributions dans Schmitter/ Lehmbruch (1979), ainsi que Lehmbruch / Schmitter (1982); Alemann (1981); Berger (1981). Pour aller plus avant: Cawson (1983), et les contributions de K. Armingeon et W. Bonß / R. Steiert in : Sozialwissenschaftliche Informationen, $11 / 1,1982$, p. 14-22 et 45-50. Voir aussi les recueils de données proposés récemment dans Flora et al. (1982); Flora / Heidenheimer (1981) ; Heidenheimer et al. (1983 [1976]) ; Alber (1982), ainsi que Brown / Browne (1968) et la discussion proposée dans Zapf (1974-1975).

k. Les termes de « corporation » ou « organisation corporative » ne se rapportent en allemand, à la différence de l'anglais, pas seulement à des entreprises ou conglomérats privés, mais également à l'organisation de certains secteurs professionnels et économiques sous surveillance étatique, ou créés sur initiative étatique. On les caractérise de «neoständisch " (néocorporatiste) ou « berufsständisch » (organisés par métiers) s'ils sont obligatoires et s'ils contrôlent l'accession à 
ces professions, c'est-à-dire s'ils tentent de rétablir sur un mode nouveau les privilèges des guildes qui avaient été supprimés lors de l'instauration de la liberté des activités commerciales et industrielles (Gewerbefreiheit) [Note de l'auteur].

1. Le « cépalisme» (cepalismo en espagnol, du nom de la CEPAL ou «Comisión Económica para América Latina y el Caribe », la commission économique régionale pour l'Amérique latine et les Caraïbes, fondée en 1948 par l'ONU) désigne une théorie et une conception économique latinoaméricaine, une variante de keynésianisme, suite aux recommandations de la commission qui a formulé des stratégies de réforme fondées essentiellement sur une conception de l'émancipation socio-économique (les propositions de réformes visaient à accélérer et approfondir l'industrialisation, à promouvoir le changement structurel dans les économies latinoaméricaines, avec une réforme agraire avant tout, à diversifier la structure de production et des biens de consommation, à promouvoir des exportations, et en particulier en vue d'une expansion des marchés) [N.d.T.].

47. Voir Manoïlescu (1934), (1937) et (1929). Sur son influence sur Raúl Prebisch et la fondation de la CEPAL, voir Love (1980), notamment p. 61 sq.

48. Schmitter (1979a), p. 13.

49. Schmitter (1979b), p. 63-65, 93 ; pour de plus amples développements, voir Schmitter (1983) ainsi que les débats au sein de l'European Group of Organizational Studies (Florence, 1983).

50. Schmitter (1979a), p. 20-22, 33-42 ; Lehmbruch (1979a), p. 54.

51. Ainsi Lehmbruch (1979a), p. 53 sq. Lehmbruch (1979b), p. 149-152 ; Beyme (1979), p. 229-262 ; Beyme (1984).

52. Beyme (1984).

53. Salisbury (1979) parvient à la conclusion très formelle qu'il n'y a pas du tout de corporatisme aux États-Unis. T. J. Pempel et K. Tsunekawa (Pempel / Tsunekawa [1979]), essaient d'intégrer l'absence d'un mouvement ouvrier organisé en tant qu'élément modifiant la puissance. Récemment, voir également Cox / Hayward (1983).

54. Schmitter (1979a), p. 40.

55. À l'opposé d'une conceptualisation relâchée, Maier (1981). U. Nocken donne des titres nouveaux à des phases bien connues de l'histoire allemande: state corporatism jusqu'en 1850, pluralism pour la période 1850-1914, state corporatism pour les périodes 1914-1924 et 1933-1945, societal corporatism pour la période 1924-1933 et à partir de 1945 (dans ce cas, mélangé avec du state corporatism) ; Nocken (1978). W. Abelshauser date de la fin du Reich impérial d'avant 1914 l'éclosion d'un corporatisme (societal) allemand (qui s'épanouit pleinement durant la République de Weimar avant d'être déformé en un sens autoritaire sous le régime national-socialiste); Abelshauser (1981), p. 316.

56. Cela tient peut-être aussi, en partie, au «décret sur la pureté » des théoriciens : l'établissement de la relation tripartite constitutive du corporatisme coïncide généralement avec la fin de la transition vers le «capitalisme organisé » (dans un contexte conceptuel tout différent).

\section{INDEX}

Mots-clés : capitalisme, corporatisme, concepts

Schlüsselwörter : Kapitalismus, Korporatismus, Konzepte 


\section{AUTEURS}

\section{HANS-JÜRGEN PUHLE}

Hans-Jürgen Puhle est professeur émérite de sciences politiques à l'Université Goethe de Francfort-sur-le-Main. Pour plus d'informations voir la notice suivante. 